\title{
Comparative evaluation of the effects of pesticides in acute toxicity luminescence bioassays
}

\author{
Amadeo R. Fernández-Alba ${ }^{\text {a,* }}$, M.D. Hernando Guil ${ }^{\mathrm{a}}$, \\ Gema Díaz López $^{\mathrm{c}}$, Yusuf Chisti ${ }^{\mathrm{b}}$

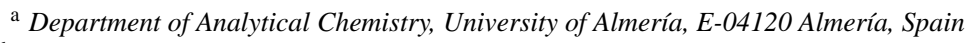 \\ ${ }^{\mathrm{b}}$ Department of Chemical Engineering, University of Almería, E-04120 Almería, Spain \\ ${ }^{\mathrm{c}}$ Ito Carlos III, Centro Nacional de Sanidad Ambiental, Ministerio de Sanidad y Consumo, E-28200 Majadahonda, Madrid, Spain
}

Received 31 May 2001; received in revised form 12 September 2001; accepted 24 September 2001

\begin{abstract}
Acute toxicity of pesticides in water was assessed singly and in mixtures using the responses of the luminescent bacterium Vibrio fischeri $\left(\right.$ BioTox $\left.^{\mathrm{TM}}\right)$, the aquatic invertebrate Daphnia magna $\left(\right.$ Daphtoxkit $\left.^{\mathrm{TM}}\right)$, and the MitoScan ${ }^{\mathrm{TM}}$ assay. The latter utilized fragmented mitochondria to enzymatically convert $\beta$-nicotinamide adenine dinucleotide (NADH) to its oxidized form, $\mathrm{NAD}^{+}$. The rate of the conversion being sensitive to type and concentration of toxicants. The pesticides tested were Carbofuran (2,3-dihydro-2,2-dimethylbenzofuran-7-yl methylcarbamate), Cyromazine ( $N$-cyclopropyl-1,3,5-triazine-2,4,6-triamine), Fenamiphos (ethyl 4-methylthio-m-tolyl isopropylphosphoramidate), and Formetanate (3-dimethylaminomethyleneiminophenyl methylcarbamate). The toxicity bioassays were characterized in terms of relative sensitivity, reproducibility, range of the linear response, and the ability to reveal synergistic/antagonistic interactions among toxicants. The D. magna assay was the most sensitive and best able to detect toxic interactions of mixtures. Also, unlike the other assays used, the response of the daphnid system was linear over a 10 -fold change in pesticide concentration. Relative to the BioTox ${ }^{\mathrm{TM}}$, the MitoScan ${ }^{\mathrm{TM}}$ was 2 - to 11 -fold more sensitive for the compounds and mixtures tested. The $\mathrm{EC}_{50}$ reproducibility of all tests was within $\pm 20 \%$ coefficient of variation; however, the lowest observable effect concentration (LOEC) were only reproducible to $\pm 35 \%$ on average. Cyromazine was the least toxic of the pesticides tested. To test the predictive value of the concept of concentration addition, toxicities of binary and quaternary mixtures of four different pesticides were analyzed. Synergistic/antagonistic responses were most frequently observed in testing with D. magna. Synergistic/antagonistic effects were seen only in 25 and $50 \%$ of the cases with the BioTox ${ }^{\mathrm{TM}}$ and the MitoScan ${ }^{\mathrm{TM}}$ assays, respectively. (C) 2002 Elsevier Science B.V. All rights reserved.
\end{abstract}

Keywords: Vibrio fischeri; Daphnia magna; MitoScan ${ }^{\mathrm{TM}}$; Carbofuran; Cyromazine; Fenamiphos; Formetanate; Mixtures; $\mathrm{EC}_{50}$; LOEC; Toxicity

\section{Introduction}

Hundreds of new chemicals are introduced into commerce every year and may eventually end up in the environment. Many of these new and existing

\footnotetext{
* Corresponding author.

E-mail address: amadeo@ual.es (A.R. Fernández-Alba).
}

compounds are toxic to the biosphere. Compounds that are deliberately released into the environment in large amounts are of special concern, because of the nature of their application. Pesticides used in agriculture are examples of such compounds. Pesticides are necessarily toxic, at least to a part of the biosphere, as a requirement of their function; however, a broader ecotoxicity of a compound and its persistence are 
matters of extreme public concern. Pesticides are commonly encountered singly and as mixtures in drinking water, rivers, lakes and other aquatic bodies. In such instances of contamination, the quantification of the concentration of the contaminant(s) is plainly insufficient unless the concentration can be satisfactorily related to the toxicity. In addition to having an impact on the discharge regulations, the toxicity considerations are important because a compound's toxicity can affect its rate of degradation in natural and manmade waste treatment processes [1].

The toxicity of pesticide contaminated effluent depends on the amounts and types of the individual pesticides present; however, even for pure compounds, concentration-toxicity relationships are complex. Mixtures of compounds may pose bigger problems because the toxicity of a mixture is not easily linked to individual toxicities of components in the mixture; synergistic and antagonistic response are know to occur [2-4]. Thus, for predicting the impact of a wastewater stream on the ecology of a receiving body such as a biological wastewater treatment facility, or a lake, the toxicity of the contaminated water needs to be determined with single and multiple contaminants and with a selection of assay methods. Multiple bioassays are necessary because no single method can reveal the possible interactions of a toxicant with the great variety of biochemistries of living systems. So far, toxicity of compounds has been assessed as single compounds because of a lack of standardized methods for assessing synergistic toxicity [5]; nevertheless, the existence of synergistic effects is well-known [5-9] and there is a clear need to quantify the aggregate toxic impact of pesticide mixtures. For example, a recent report of the US Geological Survey on the quality of the aquatic environment emphasized the need to consider synergistic effects of chemical mixtures on aquatic toxicity [7].

The subject of whole effluent (i.e. one containing a mix of chemicals) toxicity testing has been reviewed recently [7]. Whole effluent toxicity (WET) testing is useful for assessing the potential impact of the effluent on the biotic environment; however, the WET methods are subject to inherent variability, species differences, and response modification caused by physicochemical and biological factors in the receiving environment [7]. Variability is higher for moderately toxic effluent samples than those with low or high toxicity [7]. With regards to species, genetic differences between different populations are known to affect the toxic response. Also, differences in diet, temperature and water quality can alter the response of daphnids and other organisms used in testing [7].

Except over a narrow range of concentrations, the toxicity response plots are generally nonlinear. Nontraditional concentration response curves in which a small amount of a toxicant actually produces a beneficial response (a phenomenon known as hormesis) have been demonstrated [7]. Although, the so called beneficial response to a toxic substance is really an upset of the system relative to the control and any upset whether beneficial or not should be viewed as an unwanted abnormal effect on the ecosystem. Furthermore, the results of the laboratory toxicity tests do not necessarily correlate with the impact that an effluent has on the biosphere; nevertheless, such tests do provide an indication of the relative hazard posed by a discharge. Inferring risk from hazard is certainly not always correct [7] but is a safer approach to establish the effluent discharge guidelines.

This study presents a detailed analytical assessment of several bioassays for toxicity. Identified are important factors such as the range of linear response of the assay, the relative sensitivity, reproducibility, and the ability to discern synergistic and/or antagonistic effects of pesticide mixtures. Three rapid and relatively inexpensive toxicity bioassays were used: (i) the luminescent bacterium Vibrio fischeri; (ii) the aquatic invertebrate Daphnia magna is a preferred test system for aquatic toxicology [10]; and (iii) the MitoScan ${ }^{\mathrm{TM}}$ mitochondria-derived assay [11,12]. The toxicity end-point $\left(\mathrm{EC}_{50}\right)$ for each bioassays was determined as the concentration of a test sample that causes a 50\% reduction in the biological response studied. Acute toxicity was evaluated of water samples contaminated with various amounts of the following pesticides: Carbofuran (2,3-dihydro-2,2-dimethylbenzofuran-7-yl methylcarbamate), Cyromazine ( $N$-cyclopropyl-1, 3,5-triazine-2,4,6-triamine), Fenamiphos (ethyl 4methylthio- $m$-tolyl isopropylphosphoramidate), and Formetanate (3-dimethylaminomethyleneiminophenyl methylcarbamate). These compounds are commonly used in agricultural activities as systemic insecticide, acaricide and nematicides. Formetanate, Fenamiphos and Carbofuran are cholinesterase inhibitors and Cyromazine interferes in the growth of insects. 


\section{Materials and methods}

The following pesticides were tested singly and in mixtures: Carbofuran, Cyromazine, Fenamiphos, and Formetanate (Fig. 1). Pure pesticides (>98.0\% pure) were purchased from Riedel-de-Haën (Seelze, Germany). Stock solutions of the pesticides and mixtures were prepared in distilled water for V. fischeri and MitoScan ${ }^{\mathrm{TM}}$ assays and in culturing medium for $D$. magna assays. Mixtures of pesticides were prepared by combining various stock solutions of pure components in 1:1 ratio. When using pesticide mixtures, the concentration of the toxicant was defined as the sum of concentrations of all the pesticides in solution. In those cases, where the compounds had low solubility in water, $0.5 \% \mathrm{v} / \mathrm{v}$ of dimethyl sulfoxide (DMSO) was used to facilitate dissolution. Pesticide-free aqueous solutions of DMSO $(0.5 \% \mathrm{v} / \mathrm{v})$ exhibited no observable toxic response in the bioassays used. For $V$. fischeri assay, the osmolality of solutions was adjusted to $2 \% \mathrm{NaCl}$ for its optimal performance.

\subsection{Toxicity analyses}

\subsubsection{Bacterial bioluminescence assay}

A commercial assay marketed as BioTox ${ }^{\mathrm{TM}}$ (BioOrbit Oy, Turku, Finland) was used. The assay, based on the luminescent bacterium $V$. fischeri NRRL B-1117, is in principle similar to the better known Microtox ${ }^{\circledR}$ test [13-15]. The effect of test compounds on the luminiscent bacterium $V$. fischeri was evaluated at 5, 15 and $30 \mathrm{~min}$ of exposition determining the $\mathrm{EC}_{50}$. The bacteria were purchased as freeze-dried reagents. They were stored at $-20^{\circ} \mathrm{C}$ and rehydrated prior to testing. Light emitted from bacterium is a result of the interaction of the enzyme luciferase, reduced flavin, and a long-chain aldehyde in the presence of oxygen. The metabolic energy generated in this pathway is converted to chemical energy, through the electron transport system, into visible light. This metabolic pathway is intrinsically linked to cellular respiration, so disruption of normal cellular metabolism causes a decrease in light production. The toxicity end-point $\left(\mathrm{EC}_{50}\right)$ was determined as the concentration of a test sample that causes a $50 \%$ reduction in light output. The acute bioluminescence assay was carried out according to ISO 11348 [16]. Light production from luminescent bacteria was measured with a photomultiplier in a luminometer equipped with a constant temperature water bath $\left(15^{\circ} \mathrm{C}\right)$.

In addition to the $\mathrm{EC}_{50}$ value, the lowest observable effect concentration (LOEC) was also determined. The LOEC is analogous to the "limit of detection" of the conventional methods of analysis. In keeping with the established practice, the LOEC was obtained as follows:

LOEC $=\frac{3 S}{m}$

where $S$ and $m$ were the standard error in the intercept and the slope of the linear response-dose plot, respectively.<smiles>Nc1nc(N)nc(NC2CC2)n1</smiles>

Cyromazine<smiles>CCO[Nb](=O)(NC(C)C)c1ccc(S)c(C)c1</smiles>

Fenamiphos<smiles>CNC(=O)c1cccc(N=CC(C)C)c1</smiles>

Formetanate

Fig. 1. Structures of pesticides analyzed by toxicity bioassays: Carbofuran, Cyromazine, Fenamiphos and Formetanate. 


\subsection{Daphnid motility assay}

The toxicity of the compounds to the crustacean species (D. magna) was assessed using commercially available Daphtoxkit ${ }^{\mathrm{TM}}$ (Creasel, Belgium). The toxicity studies were performed in accordance with testing conditions prescribed by OECD Guideline 202 and ISO 6341 [17]. Acute toxicity was assessed by noting the effects of the test compounds on the motility of D. magna. This bioassay using dormant eggs (ephippia) treated as described in the standard operational procedure to induce hatching and the experiments was carried out on less than 24-h-old daphnids. The ephippia were incubated in standard freshwater at $21 \pm 1^{\circ} \mathrm{C}$ under continuous illumination of $6000 \mathrm{~lx}$. Between hatching and the test, the daphnids were fed on the dried cyanobacterium Spirulina. For the test, the animals were incubated for various periods with the specified concentrations of pesticides. All the test were conducted in the dark at a constant temperature of $20 \pm 1{ }^{\circ} \mathrm{C}$. The neonates are considered immobilized, after 24 and $48 \mathrm{~h}$ of incubation, if they lay on the bottom of the multi-well test play and do not resume swimming within $15 \mathrm{~s}$ of observation. The toxicity end-point $\left(\mathrm{EC}_{50}\right)$ was determined as the concentration estimated to immobilize $50 \%$ of the daphnids after 24 and $48 \mathrm{~h}$ of exposure.

For the daphnid test, the LOEC was determined as the concentration of a test sample that produced a $10 \%$ change in the biological response studied (motility).

\subsection{MitoScan ${ }^{\mathrm{TM}}$ mitochondrial assay}

MitoScan ${ }^{\mathrm{TM}}$ toxicity assay (BioRenewal Technologies, Madison, WI, USA) utilizes fragmented mitochondria or submitochondrial particles (SMP) that are functionally equivalent to the whole organelle. SMPs prepared from bovine heart mitochondria were stored frozen $\left(-80^{\circ} \mathrm{C}\right)$ until use.

The test monitored the appearance of $\mathrm{NAD}^{+}$via oxidation of $\beta$-nicotinamide adenine dinucleotide $(\mathrm{NADH})$. The rate of appearance of $\mathrm{NAD}^{+}$was reduced relative to controls when an interfering toxicant was present. The test was carried out in 96-well microplates using a volume of $300 \mu \mathrm{l}$ per well. The final concentrations in the test medium were: $50 \mathrm{mM}$ HEPES buffer, pH 7.5, $6 \mathrm{mM} \mathrm{MgSO}_{4}$, and $0.017 \mathrm{mg} / \mathrm{ml}$ SMP protein. The reaction was initiated by adding $0.137 \mathrm{mM}$ of NADH. The appearance of $\mathrm{NAD}^{+}$with time was monitored spectrophotometrically at a wavelength of $340 \mathrm{~nm}$ (Labsystems Multiskan RC microplate reader). For each test, vial containing a given dilution of the toxicant, the appearance of $\mathrm{NAD}^{+}$was monitored. A least-square fit of the linear portion of the data provided the slope, i.e. the rate of generation of $\mathrm{NAD}^{+}$. The rates obtained in the various vials were plotted against the concentration of the pesticide to obtain a dose-response curve.

\section{Results and discussion}

The toxicity end-point was determined as $\mathrm{EC}_{50}$, i.e. the effective concentration required for a $50 \%$ change in the biological response studied (bioluminescence, motility, enzyme activity). The pesticides used covered different classes of compounds with different modes of pesticide action, thus the various toxicity assays could be assessed for their abilities to respond to a range of possible interactions of the toxicant with the assay system. The assays used were relatively rapid and simple, yet covered a broad range of life biochemistries, including two whole organism assays $\left(\right.$ BioTox $^{\mathrm{TM}}$ and Daphtoxkit ${ }^{\mathrm{TM}}$ ) and a multi-enzyme assay based on fragmented mitochondria. The organisms used included a prokaryote ( $V$. fischeri) and a multi-cellular eukaryote, D. magna. The latter was biochemically the most complex test system used and was also found to be the most sensitive.

The $\mathrm{EC}_{50}$ values obtained for various compounds, mixtures, and assays are noted in Table 1 which also lists the coefficient of variation for the measurements. In both the BioTox ${ }^{\mathrm{TM}}$ and the daphnid tests, the $\mathrm{EC}_{50}$ values declined with increasing duration of exposure $(5,15,30 \mathrm{~min}$ for V. fischeri; 24 and $48 \mathrm{~h}$ for D. magna) to pesticides. This is understandable, as a larger amount of a toxicant is needed to produce the same response over a shorter incubation period. The D. magna test was by far the most sensitive; the $\mathrm{EC}_{50}$ values obtained with this test were in the parts per billion range, except for the relatively nontoxic Cyromazine. D. magna was sensitive to $0.1 \mathrm{ppb}$ total pesticide concentration in quaternary mixtures. The $\mathrm{EC}_{50}$ values determined with the BioTox ${ }^{\mathrm{TM}}$ and the MitoScan ${ }^{\mathrm{TM}}$ assays were in the milligram per liter range; the MitoScan ${ }^{\mathrm{TM}}$ test was 2- to 11-fold more 
Table 1

$\mathrm{EC}_{50}$ values $(\mathrm{mg} / \mathrm{l} \mathrm{CV} \%)$ for single pesticide and mixtures of pesticides analyzed by toxicity bioassays for different exposure times

\begin{tabular}{|c|c|c|c|c|c|c|}
\hline \multirow[t]{2}{*}{ Pesticides and mixtures } & \multicolumn{3}{|l|}{ BioTox $^{\mathrm{TM}}$} & \multirow{2}{*}{$\frac{\text { MitoScan }^{\mathrm{TM}}}{40(\mathrm{~min})}$} & \multicolumn{2}{|c|}{ DaphtoxKit $^{\mathrm{TM}}$} \\
\hline & $5(\min )$ & $15(\min )$ & $30(\min )$ & & $24(\mathrm{~h})$ & 48 (h) \\
\hline Formetanate & $18.8(33)$ & $7.4(37)$ & $4.8(40)$ & $3.3(6)$ & $0.1(22)$ & $0.077(34)$ \\
\hline Carbofuran & $33.3(5)$ & $31.2(7)$ & $28.9(17)$ & $7.6(30)$ & $0.096(16)$ & $0.018(20)$ \\
\hline Cyromazine & None & None & None & $26.1(26)$ & None & $10.7(20)$ \\
\hline Fenamiphos & $38.4(14)$ & $35.1(22)$ & $33.2(22)$ & $2.9(15)$ & $0.025(15)$ & $0.005(5)$ \\
\hline Formetanate-Carbofuran & $31.1(14)$ & $16.7(18)$ & $11.4(20)$ & $3.6(6)$ & $0.1(18)$ & $0.03(20)$ \\
\hline Formetanate-Fenamiphos & $27.7(20)$ & $11.9(17)$ & $8.7(20)$ & $9.5(30)$ & 0.09 (18) & $0.001(30)$ \\
\hline Carbofuran-Cyromazine & $61.3(23)$ & $50.8(13)$ & $46.5(26)$ & $5.6(20)$ & $0.05(18)$ & $0.005(20)$ \\
\hline $\begin{array}{l}\text { Formetanate-Carbofuran- } \\
\text { Cyromazine-Fenamiphos }\end{array}$ & $22.3(12)$ & $7.4(25)$ & $4.4(20)$ & $22.8(14)$ & $0.01(18)$ & $1 \times 10^{-5}(10)$ \\
\hline
\end{tabular}

sensitive than the BioTox ${ }^{\mathrm{TM}}$ for the compounds and mixtures tested.

For pure compounds in the BioTox ${ }^{\mathrm{TM}}$ test, toxicity followed the order, Cyromazine, Fenamiphos, Carbofuran, and Formetanate, irrespective of the duration of the exposure. This pattern was different for the other assays used; however, in all the cases Cyromazine proved to be the least toxic of the pesticides tested. The $\mathrm{EC}_{50}$ values for this compound were 10.7 and $26.1 \mathrm{mg} / \mathrm{l}$ with the daphnid and the MitoScan ${ }^{\mathrm{TM}}$ assays, respectively. No toxic effect was detected for Cyromazine with the BioTox ${ }^{\mathrm{TM}}$ test. In fact, the light emission was measurably enhanced during contact with Cyromazine, possibly because it was being consumed as a nutrient by the cells [9]. Because the highest Cyromazine concentration in the incubation vial was $50 \mathrm{mg} / \mathrm{l}$, it may be concluded that the BioTox ${ }^{\mathrm{TM}}$ $\mathrm{EC}_{50}$ value for Cyromazine exceeds $50 \mathrm{mg} / \mathrm{l}$.
The LOEC data are shown in Table 2. As expected, the $\mathrm{EC}_{50}$ values were always greater than the corresponding LOECs: 10 - to 150 -fold greater for the BioTox $^{\mathrm{TM}}$; 2- to 30-fold greater for the MitoScan ${ }^{\mathrm{TM}}$; and 5- to 100-fold greater for the D. magna test. In addition to the $\mathrm{EC}_{50}$ value, a consideration of the LOECs can be useful in establishing the effluent discharge limits. If the LOEC is much less than the $\mathrm{EC}_{50}$, the prudent practice is to base the limit on the LOEC value. The $\mathrm{EC}_{50}$ is merely an index of relative toxicity whereas the LOEC provides compound-specific data that is more directly relevant.

Compared to conventional chemical analyses, toxicity bioassays are much less reproducible. Variability within a factor of two is generally considered acceptable for toxicity results on a given effluent and using a given toxicity test [7]. In the present work, the $\mathrm{EC}_{50}$ reproducibility was within $\pm 20 \%$ (coefficient of

Table 2

LOEC values $(\mathrm{mg} / \mathrm{l} \mathrm{CV} \%)$ and their coefficients of variation for single and mixtures pesticides analyzed by various toxicity bioassays for different exposure times

\begin{tabular}{|c|c|c|c|c|c|c|}
\hline \multirow[t]{2}{*}{ Pesticides and mixtures } & \multicolumn{3}{|l|}{ BioTox $^{\mathrm{TM}}$} & \multirow{2}{*}{$\frac{\text { MitoScan }^{\mathrm{TM}}}{40 \text { (min) }}$} & \multicolumn{2}{|l|}{ Daphtoxkit $^{\mathrm{TM}}$} \\
\hline & $5(\min )$ & 15 (min) & $30(\min )$ & & $24(\mathrm{~h})$ & 48 (h) \\
\hline Formetanate & $1.4(14)$ & $1.2(9)$ & $1.2(12)$ & $0.86(25)$ & & $0.01(30)$ \\
\hline Carbofuran & $2.4(30)$ & $1.38(21)$ & $1.5(16)$ & $0.24(30)$ & $0.018(30)$ & $0.0018(28)$ \\
\hline Cyromazine & None & None & None & $3(25)$ & None & $1.07(30)$ \\
\hline Fenamiphos & $1.5(35)$ & $1.38(35)$ & $1.6(30)$ & $0.3(26)$ & $9 \times 10^{-4}(30)$ & $9 \times 10^{-4}(30)$ \\
\hline Formetanate-Carbofuran & $1.4(24)$ & $1.7(35)$ & $1.7(35)$ & $1.7(30)$ & $0.01(40)$ & $0.01(40)$ \\
\hline Formetanate-Fenamiphos & $0.18(30)$ & $0.18(30)$ & $0.2(21)$ & $1.26(30)$ & $0.001(40)$ & $0.001(40)$ \\
\hline Carbofuran-Cyromazine & $0.47(30)$ & $0.21(30)$ & $0.26(30)$ & $3.2(30)$ & $0.001(40)$ & $0.001(40)$ \\
\hline $\begin{array}{l}\text { Formetanate-Carbofuran- } \\
\text { Cyromazine-Fenamiphos }\end{array}$ & $0.31(40)$ & $0.23(30)$ & $0.25(30)$ & $10(30)$ & $1 \times 10^{-4}(31)$ & $1 \times 10^{-5}$ \\
\hline
\end{tabular}


variation) for all the tests (Table 1). The reproducibility data given are based on three replicates each for the BioTox ${ }^{\mathrm{TM}}$ and the MitoScan ${ }^{\mathrm{TM}}$ tests; the $D$. magna data are for four replicates. Generally the $\mathrm{EC}_{50}$ value was more reproducible than the LOEC. The average reproducibility of the LOEC data was 27,28 , and $34 \%$ (coefficient of variation) for the BioTox ${ }^{\mathrm{TM}}$, the MitoScan ${ }^{\mathrm{TM}}$, and the D. magna assays. For the $\mathrm{EC}_{50}$ data, the average reproducibility was 20,18 , and $19 \%$ for the BioTox ${ }^{\mathrm{TM}}$, the MitoScan ${ }^{\mathrm{TM}}$, and the D. magna measurements. The generally better than normal reproducibility is attributable to the fact that the 'whole effluent' used was relatively simple and clean (i.e. solution of one to four pure compounds in ultrapure water).

Except for the BioTox ${ }^{\mathrm{TM}}$ assay, the dose-response relationships were generally logarithmic both for the pure compounds and the mixtures tested. The linear response range was generally quite narrow for the BioTox $^{\mathrm{TM}}$ and the MitoScan ${ }^{\mathrm{TM}}$ assays but at least 10 -fold for the D. magna test. Some representative data on the linear ranges are given in Table 3 for various test compounds. The narrow range of linear response for the BioTox ${ }^{\mathrm{TM}}$ and the MitoScan ${ }^{\mathrm{TM}}$ suggest the involvement of multiple enzymes in determining the toxicity response of those tests. A larger range of linear response at extremely low concentrations of toxicants (generally $<1 \mathrm{mg} / \mathrm{l}$ ) suggests that the response of $D$. magna is likely associated with one enzyme necessary for some critical metabolic function. Of course, the one enzyme being affected could be different for the different toxicants. The data in Table 3 were measured at the following range of concentrations: $1.50-50.00 \mathrm{mg} / \mathrm{l}$ for the BioTox $^{\mathrm{TM}}$

Table 3

Typical linear response range of the pesticides analyzed by toxicity bioassays

\begin{tabular}{llll}
\hline Pesticides & \multicolumn{2}{l}{ Linear response range $(\mathrm{mg} / \mathrm{l})$} \\
\cline { 2 - 4 } & BioTox $^{\mathrm{TM}}$ & MitoScan $^{\mathrm{TM}}$ & Daphtoxkit $^{\mathrm{TM}}$ \\
\hline Formetanate & $1.60-25.75$ & $0.50-4.10$ & $0.10-1.00$ \\
Fenamiphos & $6.50-52.00$ & $0.50-2.20$ & $0.096-0.96$ \\
Carbofuran & $4.00-35.25$ & $0.70-3.10$ & $0.018-0.18$ \\
$\begin{array}{l}\text { Cyromazine } \\
\begin{array}{l}\text { Quaternary } \\
\text { mixture }\end{array}\end{array}$ & Non toxic & $1.06-4.60$ & $1.07-10.07$ \\
\hline
\end{tabular}

(except for cyromazine when the concentration range was $3-212 \mathrm{mg} / \mathrm{l}) ; 0.5-100.0 \mathrm{mg} / \mathrm{l}$ for the MitoScan ${ }^{\mathrm{TM}}$ $(1-10 \mathrm{mg} / \mathrm{l}$ when Cyromazine was used); and $0.1 \mathrm{ppb}$ to $1 \mathrm{mg} / \mathrm{l}$ for D. magna $(1-10 \mathrm{mg} / \mathrm{l}$ when Cyromazine was used).

\subsection{Toxicity of pesticide mixtures}

No method exists for relating the toxicities of individual components of a mixture to the mixture's toxicity, except when synergistic and antagonistic effects are absent. In the latter event, when compounds act independently, the toxicities are sometimes additive. When this occurs, a mixture's toxicity may be estimated as a weighted sum of toxicities of the individual components present $[8,16]$. Another approach to estimating the mixture toxicity when the components are noninteracting is the use of toxicity units. A toxicity unit is the concentration of a component $i$ divided by its $\mathrm{EC}_{50}$ value; thus,

$\mathrm{TU}_{i}=\frac{C_{i}}{\mathrm{EC}_{50_{i}}}$

The mixture toxicity is then given by

$\mathrm{TU}_{\mathrm{M}}=\sum_{i=1}^{n} \mathrm{TU}_{i}$

If the measured toxicity is greater or less than the predicted value, synergistic and antagonistic interactions among components are revealed; however, it is impossible to say a priori whether the $\mathrm{TU}_{\mathrm{M}}$ calculated with Eq. (3) will be equal to, greater than, or less than the measured toxicity of the mixture. The toxicity unit values of the mixtures and components were calculated and compared with the measured mixture toxicity to reveal the interactions shown in Table 4. Note that $\mathrm{TU}_{i}$ and $\mathrm{EC}_{50}$ are not comparable quantities as one is dimensionless and the other is a concentration. Similarly, the toxicity units of a mixture cannot be compared to the $\mathrm{EC}_{50}$ value for the mixture. As shown in Table 4, synergistic/antagonistic responses were most frequently observed in testing with D. magna; in fact all mixtures tested produced such responses with this test. With the same mixtures, synergistic/antagonistic effects were seen only in 25 and $50 \%$ of the cases with the BioTox ${ }^{\mathrm{TM}}$ and the MitoScan ${ }^{\mathrm{TM}}$ assays, respectively. 
Table 4

Interactions of toxicity response

\begin{tabular}{|c|c|c|c|}
\hline \multirow[t]{2}{*}{ Mixture of pesticides } & \multicolumn{3}{|l|}{ Bioassays } \\
\hline & BioTox $^{\mathrm{TM}}$ & MitoScan $^{\mathrm{TM}}$ & Daphtoxkit $^{\mathrm{TM}}$ \\
\hline Formetanate-Carbofuran & Additive & Additive & Antagonistic \\
\hline Formetanate-Fenamiphos & Additive & Antagonistic & Synergistic $^{\mathrm{a}}$ \\
\hline Carbofuran-Cyromazine & Additive & Additive & Synergistic $^{\mathrm{a}}$ \\
\hline $\begin{array}{l}\text { Formetanate-Carbofuran- } \\
\text { Cyromazine-Fenamiphos }\end{array}$ & Synergistic & Antagonistic & Synergistic \\
\hline
\end{tabular}

${ }^{\mathrm{a}}$ The observed behavior depended on the duration of exposure ( 24 or $48 \mathrm{~h}$ ); the response shown is for $48 \mathrm{~h}$ exposure. For $24 \mathrm{~h}$ exposure, the Formetanate-Fenamiphos response was antagonistic and the Carbofuran-Cyromazine response was additive.

As noted above, no methods are currently available for predicting the toxicity response generated by a mixture of compounds from toxicity data for individual components of the mixture; however, predictive methods can be developed, at least in principle. Attaining a predictive capability requires a detailed knowledge of the kinetics of all essential metabolic reactions of a given biosystem and a quantitative understanding of how the individual toxicants interact with the various metabolic enzymes and intermediates. An understanding of the mutual chemical interaction of the compounds irrespective of the biosystems is also necessary. The metabolic network could then be modeled mathematically and such models could be used to simulate the effects of any combination of type, number, and quantity of toxicants on the response of the biosystem. Considering the already available capacity for modeling complex networks (e.g. ecosystems, the global environment, and indeed many aspects of metabolism), metabolic modeling for predicting the toxicity response is undoubtedly feasible at least for the simpler systems and the known toxicants.

\section{Conclusions}

Toxicity is a biological response and this needs to be taken into account in selection and usage of toxicity assays. As documented here, different pesticides in mixtures interact to produce synergistic and antagonistic effects on toxic response. Of the three toxicity assays evaluated, the D. magna is clearly the best in discerning a toxic effect. This assay is by far the most sensitive and it is good at detecting toxicity synergisms. The $\mathrm{EC}_{50}$ reproducibility of the D. magna assay is comparable to that of the other tests examined. Also, the daphnid test has a significantly broader range of linear response than the other assays. In comparison with the BioTox ${ }^{\mathrm{TM}}$ and the MitoScan ${ }^{\mathrm{TM}}$, the D. magna assay is cumbersome and slow; however, it requires minimal initial investment, operating expense, and technology. Furthermore, the toxicity data obtained with a relatively complex aquatic species such as $D$. magna perhaps provide a more meaningful indication of aquatic toxicity of a compound than the other tests used. Toxicity testing has a clear role in safeguarding environmental quality but a considered selection of testing methods is essential for obtaining results that are relevant. Effluent discharge criteria need to consider not only the $\mathrm{EC}_{50}$ values of pure compounds, but also the data for whole effluent (i.e. synergistic effects) and the LOEC of potential pollutants.

\section{References}

[1] P.J. Allsop, Y. Chisti, M. Moo-Young, G.R. Sullivan, Biotechnol. Bioeng. 41 (1993) 572-580.

[2] M. Faust, R. Altenburger, W. Boedeker, L.H. Grimme, Bull. Environ. Contam. Toxicol. 53 (1994) 134-141.

[3] S.S. Sharma, H. Schat, R. Vooijs, L.M. Van Heerwaarden, Environ. Toxicol. Chem. 18 (2) (1999) 348-355.

[4] N. Nirmalakhandan, V. Arulgnanendran, M. Mohsin, B. Sun, F. Cadena, Water Res. 28 (1994) 543-551.

[5] J. Byron. Environm. Sci. Technol. 1 (1999) 229A.

[6] M. Faust, R. Altenburger, W. Boedeker, L.H. Grimme. Sci. Total Environ. (Suppl.) (1993) 941-952.

[7] P.M. Chapman, Environ. Toxicol. Chem. 19 (2000) 3-13.

[8] K. Christen, Environ. Sci. Technol. (5) (1999) 230A.

[9] A.R. Fernández-Alba, L. Hernando Guil, G. Díaz López, Y. Chisti, Anal. Chim. Acta 426 (2001) 289-301.

[10] S.A. Mansour (Ed.), in: Proceedings of the 3rd Congress of Toxicology in Developing Countries, Vol. 1, National Research Council, Cairo, pp. 43-74. 
[11] G.A. Blondin, L.M. Knobeloch, H.W. Read, J.M. Harkin, Bull. Environ. Contam. Toxicol. 38 (1987) 467-474.

[12] G.A. Blondin, L.M. Knobeloch, H.W. Read, J.M. Harkin, Aquatic Toxicol. Environ. Fate 11 (1989) 551-563.

[13] J.C. Chang, P.B. Taylorand, F.R. Leach, Bull. Environ. Contam. Toxicol. 26 (1981) 150-156.
[14] J.M. Ribo, K.L.E. Kaiser, Toxic. Assess. 2 (1987) 305-323.

[15] M.J. Ruiz, L. López-Jaramillo, M.J. Redondo, G. Font, Bull. Environ. Contam. Toxicol. 59 (1997) 619-625.

[16] J.M. Ribo, F. Rogers, Toxic. Assess. 5 (1990) 135-152.

[17] Herman B.W.M. Koeter, OECD Publications. OECD Guidelines for the testing of Chemicals, Daphnia sp., Acute Immobilisation Test Vol. 1, 1995. 